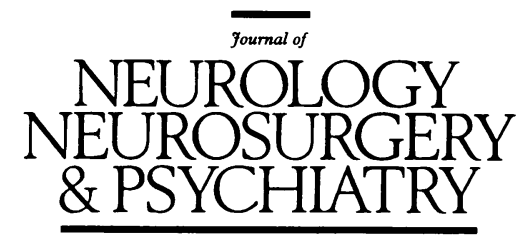

\title{
Editorial
}

\section{Pregnancy in multiple sclerosis}

Despite evidence that relapses in multiple sclerosis (MS) may be precipitated by intercurrent infection ${ }^{1}$ and administration of gamma interferon ${ }^{2}$ and that exacerbations may be partially prevented by beta interferon, ${ }^{3}$ we know little of the basic mechanisms by which demyelination may be induced or modified. There is, however, evidence from various retrospective studies of a marked amelioration of MS disease activity during pregnancy and an exacerbation in the puerperium. Other disorders of presumed immunological origin such as rheumatoid arthritis $(\mathrm{RA})^{4}$ and systemic lupus erythematosus (SLE) ${ }^{5}$ tend to stabilise during gestation and worsen in the post partum period. The mechanism of the effect of pregnancy upon these diseases has been relatively understudied as a possible source for the development of treatment to suppress disease activity.

The relationship between the course of MS and pregnancy has been addressed by a number of retrospective studies, with the attendant problems associated with patient selection and the difficulties in the assessment of relapses. In New York, Tillmann studied 52 women with MS who had 70 pregnancies and reported that the disease was exacerbated in the puerperium in 22 patients but concluded that the disease was not accelerated by pregnancy. ${ }^{6}$ Millar et al reported 45 relapses in 70 patients during 170 "pregnancy years" (nine months pregnancy and three months puerperium) and found 39 relapses in the puerperium and only six during pregnancy. ${ }^{7}$ The overall rate of relapse in "pregnancy years" $(0.265$ relapses/year) was more than twice the relapse rate for non-pregnant single or married women $(0 \cdot 104$ relapses/year). The authors found that the excess relapses occurring in the puerperium were balanced by a deficit of relapses in the following non-pregnancy years and suggested that the puerperium caused anticipation of a relapse which would have occurred otherwise at a later date. In another UK study of 124 pregnancies, 12 relapses occurred during pregnancy and 19 in the three post-partum months; the authors did not find any evidence to support the concept of "anticipation".8 An increased risk of worsening of MS in the puerperium was noted by Ghezzi and Caputo ${ }^{9}$ but because of less frequent relapses in gestation and the post-pregnancy year (after the three months puerperium) the overall effects of pregnancy on the relapse rate and disability was not significantly different from a control group. A detailed study from Israel also reported a significant reduction of relapse rate in pregnancy particularly in the third trimester but a three times higher relapse rate than expected in the first three months of the puerperium ${ }^{10}$ In that study the total number of relapses observed in the 15 months of pregnancy and puerperium was no different from that expected on the basis of 0.28 relapses/patient /year.

From Australia, using a retrospective telephone interview of 52 women, 85 pregnancy periods (nine months of pregnancy plus six months puerperium) were analysed; there were significantly fewer relapses during the first two trimesters of pregnancy with a non-significant increase in relapse rate in the last trimester of pregnancy and the first trimester of the puerperium. ${ }^{11}$ The overall relapse rate during 85 pregnancy periods was 0.38 /year compared to 0.53 relapses/year during the non-pregnancy years suggesting that overall both pregnancy and puerperium had a beneficial effect on MS relapse rate. A similar effect on the relapse rate in the "pregnancy year" (nine months of pregnancy plus six months puerperium) in comparison to the rate outside this period was noted in an Italian study; during the last trimester of pregnancy there were no relapses in 66 pregnancies. ${ }^{12} \mathrm{~A}$ three fold increase in the relapse rate during the nine months post-partum compared to the nine months of pregnancy in 111 women has been described; ${ }^{13}$ during pregnancy there were 19 exacerbations in 191 pregnancies $(10 \%)$ but during the 9 months post-partum period there were 65 exacerbations (34\%). The greatest risk was in the three month period immediately post-partum (44/65 post-partum exacerbations). Breast feeding did not affect the relapse rate. In a small prospective study eight pregnancies were examined; there were no relapses during pregnancy but six in the puerperium. $^{14}$

In summary most studies have shown a reduction of the relapse rate during pregnancy with a total of 121 relapses in 1119 pregnancies giving a relapse rate of 0.14 per year $\{(121 / 1119) \times 1 \cdot 33\}$. During 3 months puerperium the rate was 1.0 relapses per year $\{(283 / 1119) \times 4\}$. The relapse rate for the 12 month period (pregnancy + three months puerperium) was $0.36(404 / 1119)$.

In this issue of the journal the first prospective MS pregnancy study with a significant number of patients confirms the sharp increase in the relapse rate in the first three months of the puerperium; in addition post-partum relapses were more severe than those during pregnancy. ${ }^{15}$ The finding that the relapse rate during pregnancy was slightly higher than that during the non-pregnancy years 
is somewhat surprising and at odds with most retrospective studies. The overall higher relapse rate in this study probably reflects the greater accuracy and validity of prospectively collected data. As the authors indicate, the lack of any reduction in the relapse rate during any of the trimesters of pregnancy is contrary to the concept of pregnancy induced immunosuppression.

Although it has been suggested that women with onset of illness after pregnancy were more disabled than married women without pregnancies, ${ }^{8}$ various studies have shown no overall adverse effect of pregnancy on the prognosis for long-term disability in MS despite marked changes in relapse rates in pregnancy and the puerperium. ${ }^{15-19}$ In part this is due to the recognised weak association between attack frequency and disability ${ }^{20}$ and also probably due to the balancing effects of reduced relapses in pregnancy and increased relapse rates in the puerperium. In a review of hormonal factors and susceptibility to MS a long term prospective study of the effect of pregnancy on MS disability is reported to have shown a more benign course in the pregnant cohort than in a matched group of women who decided not to become pregnant $^{21}$; the problems in matching such groups are considerable (see below).

The effect of pregnancy in RA has been more intensely studied; more than $75 \%$ of pregnant patients with RA improve starting in the first or second trimester with maximum improvement in the third trimester. ${ }^{4}$ In the weeks or months of the puerperium a disease flare-up will occur in more than $90 \%$ of these patients. ${ }^{45}$ That this modulating effect of disease activity may be due to female hormones is supported by evidence that oral contraceptives protect against disease development in RA. ${ }^{22}$ There is also the suggestion that pregnancy delays the onset of RA until the post-partum period. ${ }^{23}$ In SLE the amelioration of disease activity during pregnancy is less evident; in the post-partum period exacerbation of disease activity is more likely. ${ }^{5}$ In the animal model of demyelinating disease, experimental allergic encephalomyelitis (EAE), induced in pregnant rabbits the appearance of EAE was delayed and occurred only after delivery or after fetal resorption or abortion. The incidence of EAE was lower and the levels of antibodies to myelin basic protein were less in pregnant rabbits compared with non-pregnant controls. ${ }^{24}$

The mechanisms of the apparent beneficial effects of pregnancy in autoimmune diseases are unknown. There are problems relating in vitro laboratory and animal studies to the human maternal state. Immunomodulation rather than immunosuppression has been argued as the means by which maternal acceptance of the fetal allograft is maintained. ${ }^{25}$ This modulation may relate to the interaction of many factors including marked increases in the concentrations of the hormones, cortisol, oestrogen and progesterone and the appearance of pregnancy associated proteins including pregnancy-associated plasma proteins A B and C, pregnancy-associated alpha 2 glycoprotein, pregnancy-specific B21-glycoprotein and progesterone induced blocking factor (PIBF) among others. ${ }^{26}$ Progesterone reaches its maximum concentration of 130 $\mathrm{ng} / \mathrm{ml}$ during the 36th week of pregnancy and, although not directly immunosuppressive at this concentration, is associated with the production of a $34 \mathrm{kDa}$ protein (PIBF) from maternal lymphocytes which is capable of blocking natural killer cell cytotoxicity. ${ }^{27}$ Oestrogens have a diphasic effect on the immune system being stimulatory at low and inhibitory at high concentrations, but physiological oestrogen levels are unlikely to be of significance in immunomodulation. ${ }^{25}$ Cellular factors do not show striking changes except for consistent reports of reduc- tions in the percentages of circulating CD4 $+\mathrm{T}$ lymphocyte subsets observed as early as the fourth week of gestation. ${ }^{28}{ }^{29}$ Alteration, or lack of alteration, in $T$ lymphocyte subset counts in the peripheral blood may have little relevance to the maternal-fetal interface and hormonal effects, even with the same hormone, may vary in different anatomical situations; the complexity of relationships between hormones, target cells and intracellular events including gene activation may be only addressed by advances in molecular biology. A satisfactory explanation of the biological mechanisms for the observed variation in autoimmune disease activity during pregnancy and the puerperium is elusive. ${ }^{2125263031}$

Although the evidence from the retrospective studies indicates a protective effect of pregnancy in MS with a deleterious puerperial period, questions remain which may only be addressed by a prospective study of pregnancy in MS. The problems of retrospective assessments of relapses and disability and bias towards inclusion of patients with more active disease can only be examined by a large multicentre study. It should be noted in this respect that the general acceptance of a relationship between trauma and the occurrence of MS relapse over the last 40 years ${ }^{16}$ has only recently been contradicted..$^{32}$ It is with the aim of addressing the question of the natural history of MS during pregnancy and the post-partum period that the European Committee For Research and Treatment in MS has initiated a multicentre study (PRIMS) to collect prospectively data from $250 \mathrm{MS}$ pregnancies. The patients will be followed for two years post-partum and analysed using the EDMUS system. ${ }^{33}$ Control non-pregnant MS women will not be assessed because of the insuperable problems of matching; women with MS who decide to become pregnant are likely to have less active disease than those who decide to the contrary. It is likely that beta interferon will be of particular value in the immediate post-partum period ${ }^{34}$; for this reason it would be worthwhile collecting prospective data before beta interferon becomes available in Europe. Neurologists who wish for further information about the study should contact the undersigned or one of the local coordinators (see appendix).

In addition to a clinical study of pregnancy in $\mathrm{MS}$ it is clear that work towards improved understanding of the mechanisms of immunomodulation in pregnancy may be the basis for the development of effective treatments to suppress disease activity in many autoimmune diseases.

M HUTCHINSON

Department of Neurology, St Vincent's Hospital, Elm Park, Dublin, 4. Ireland

1 Sibley WA, Bamford CR, Clark K. Clinical viral infections and multiple

2 Panitch HS, Bever CT, Katz E, Johnson KP. Treatment of multiple sclerosis with gamma interferon: exacerbations associated with activation of the immune system. Neurology 1987;37:1097-1102.

3 The IFNB Multiple Sclerosis Study Group. Interferon beta-1b is effective in relapsing-remitting multiple sclerosis 1 . Clinical results of a multicenter, randomised, double-blind, placebo-controlled trial. Neurology 1993; 43:655-61.

4 Persellin RH. The effect of pregnancy on rheumatoid arthritis. Bull Rheum Dis 1977;27:922-7.

5 Cecere FA, Persellin RH. The interaction of pregnancy and the rheumatic diseases. Clin Rheum Dis 1981;7:747-68.

6 Tillman A. The effect of pregnancy on multiple sclerosis and its management. Res Publ Assoc Res Nerv Ment Dis 1950;28:548-82.

7 Millar JHD, Allison RS, Cheesman EA, Merrett JD. Pregnancy as a factor influencing relapse in disseminated sclerosis. Brain 1959;82:417-26.

8 Schapira K, Poskanzer DC, Newell DJ, Miller H. Marriage, pregnancy and multiple sclerosis. Brain 1966;89:419-28.

9 Ghezzi A, Caputo D. Pregnancy: a factor influencing the course of multiple sclerosis? Eur Neurol 1981;20:517-9.

10 Korn-Lubetzki I, Kahona E, Cooper G, Abramsky D. Activity of multiple sclerosis during pregnancy and the puerperium. Ann Neurol 1984;16:229-31.

11 Frith JA, McLeod JG. Pregnancy and multiple sclerosis. $\mathcal{f}$ Neurol Neurosurg Psychiatry 1988;51:495-8.

12 Bernardi S, Grasso MG, Bertollini R, Orzi F, Fieschi C. The influence of 
pregnancy on relapses in multiple sclerosis: a cohort study. Acta Neurol Scand 1991;84:403-6.

13 Nelson LM, Franklin GM, Jones MC. Risk of multiple sclerosis exacerbation during pregnancy and breast feeding. $\mathcal{F A M A} 1988 ; 259: 3441-3$.

14 Birk K, Ford C, Smeltzer S, Ryan D, Miller R, Rudick RA. The clinical course of multiple sclerosis during pregnancy and the puerperium. Arch Neurol 1990;47:738-42.

15 Roullet E, Verdier-Taillefer M-H, Amarenco P, Gharbi G, Alperovitch A, Marteau R. Pregnancy and multiple sclerosis: a longitudinal study of 125 remittent patients. F Neurol Neurosurg Psychiatry 1993;56:1062-5

16 McAlpine D, Compston N. Some aspects of the natural history of disseminated sclerosis. Quart $\mathcal{F}$ Med 1952;21:135-67.

17 Poser S, Poser W. Multiple sclerosis and gestation. Neurology 1987;33: 1422-27.

18 Thompson DS, Nelson LM, Burns A, Burks JS, Franklin GM. The effects of pregnancy in multiple sclerosis: a retrospective study. Neurology 1986;36:1097-9.

19 Weinshenker BG, Hader W, Carriere W, Baskerville J, Ebers GC. The influence of pregnancy on disability from multiple sclerosis: a population-based study in Middlesex County, Ontario. Neurology 1989, 39:1438-40.

20 Weinshenker BG, Bass B, Rice GPA, Noseworthy J, Carriere W, Baskerville J, Ebers GC. The natural history of multiple sclerosis: a geographically based study. 2. Predictive value of the early clinical course. Brain 1989;112:1419-28.

21 Duquette $P$, Girard $M$. Hormonal factors in susceptibility to multiple sclerosis. Current Opinion in Neurology and Neurosurgery 1993;6. sclerosis.

22 Hazes JMW, Van Zeben D. Oral contraception and its possible protection against rheumatoid arthritis. Ann Rheum Dis 1991;50:72-4.

23 Del Junco DJ, Annergers JF, Coulam CB, Luthra HS. The relationship between rheumatoid arthritis and reproductive function. $\mathrm{Br} \mathcal{F}$ Rheumatol 1989;28(Suppl 1):33

24 Evron S, Brenner T, Abramsky O. Suppressive effect of pregnancy on the development of experimental allergic encephalomyelitis in rabbits. $A m \mathcal{F}$ Reprod Immunol 1984;5:109-13.

25 Hegde UC. Immunomodulation of the mother during pregnancy. Med Hypotheses 1991;35:159-64.

26 Pope RM. Immunoregulatory mechanisms present in the maternal circulation during pregnancy. Baillieres Clin Rheumatol 1990;4:33-52.

27 Szekeres-Bartho J, Varga P, Pejtsik B. Elisa test for the detection of an immunological blocking factor in human pregnancy serum. 7 Reprod immunological blocking

28 Degenne D, Canepa S, Lecomte C, Renoux M, Bardos P. Serial study of T-lymphocyte subsets in women during very early pregnancy. Clin Immunol Immunopathol 1988;48:187-91.

29 Gargiulo P, Di Mario U, Dotta F, Kennedy R, Guy K, Pachi A, Fallucca F. Activated $T$ cells in normal pregnant women and neonates. Eur $\mathcal{F}$ Obstet Gynecol Reprod Biol 1986;23:127-35.

30 Lahita RG. Sex hormones and the immune system-part 1. Human data. Baillieres Clin Rheumatol 1990;4:1-12.

31 Ansar Ahmed S, Talal N. Sex hormones and the immune system-part 2. Animal data. Baillieres Clin Rheumatol 1990;4:13-31.

32 Sibley WA, Bamford CR, Clark K, Smith MS, Laguna JF. A prospective study of physical trauma and multiple sclerosis. $f$ Neurol Neurosurg
Psychiatry 1991;54:584-9.

33 Confavreux C, Compston DAS, Hommes OR, McDonald WI, Thompson AJ. EDMUS, a European database for multiple sclerosis. f Neurol Neurosurg Psychiatry 1992;55:671-6.

34 Arnason BGW. Interferon beta in multiple sclerosis. Neurology 1993;43: 641-3.

\section{Appendix}

PRIMS: Coordinating committee: A Alperovitch (statistician), A Biron, H Carton, C Confavreux, OR Hommes, MB D'Hooghe, $M$ Hutchinson.

LOCAL COORDINATORS: Austria: Dr Harald Kollegger, Neurological Clinic, University of Vienna, Wahringer Gurtel 18-20, A-1090, Wien. Belgium: Professor Herwig Carton, Department of Neurology, University Hospitals KU Leuven, B-3000 Leuven. Dr M Bieke D'Hooghe, Centre Nat de la Sclerose en Plaques, Vanheylenstraat 16, B-1820 Melsbroek. Denmark: Dr Anne Heltberg, Department of Neurology, Amtssygehuset Roskilde, Koegevej 7-13, P O Box 247, 4000 Roskilde. Dr Hans Jakob Hansen, Department of Neurology, Arhus Kommunehospital, 8000 Arhus C. France: Professor Christian Confavreux, Hopital Neurologique, 59 Boulevard Pinel, BP Lyon Montchat, 69003 Lyon. Germany: Professor Judith Haas, Department of Neurology, Medizinische Akademie Magdeburg, Leipziger Str 44, D-3090 Magdeburg. Ireland: Dr Michael Hutchinson, St Vincent's Hospital, Elm Park, Dublin 4. Italy: Professor Giancarlo Comi, Department of Neurology, Scientific Institute San Raffaele, Via Olgettina 60, 20132 Milano; Dr Maria Pia Amato, Dipart di Scienze Neurol, Universita di Firenze, Ospedale di Carreggi, Viale Morgagni 85, 50134 Firenze. The Netherlands: Professor Otto Hommes, Institute of Neurology, University Hospital Nijmegan, Reinier Postlaan 4, P.O. Box 9101, 6500 HB Nijmegan. Portugal Dr Joao Correia de Sa, Department of Neurology, Hospital de Santa Maria, Av Egas Moniz, 1600 Lisboa. Spain: Dr O Fernandez, Servicio de Neurologia, Hospital Regional de Malaga Carlos Haya, Avda Carlos Haya, 29010 Malaga. Sweden: Dr Oluf Andersen, Department of Neurology, Sahlgren Hospital, S41345, Goteburg. Switzerland: Dr Ludwig Kappos. Neurol Universitatsklinik Kantonsspital, Petersgraben 4, CH-Basel. United Kingdom: Dr Alan Thompson, Institute of Neurology, The National Hospital, Queen Square, London WC1N 3BG; Dr David Bates, Department of Neurology, The Royal Infirmary, Queen Victoria Road, Newcastle upon Tyne, NE1 4LP; Dr Ian Bone, Institute of Neurological Sciences, Southern General Hospital, 1345, Govan Road, Glasgow G51 4TF; Dr Stanley Hawkins, Department of Neurology, Royal Victoria Hospital, Grosvenor Rd, Belfast, BT12 6BJ, N Ireland. 